\title{
Perceptions on activity behavior during the COVID-19 pandemic "second wave" among US adults: results of a short online survey
}

\author{
Emerson Sebastião ${ }^{1}\left[\right.$ Michelle Steffens $^{1} \cdot$ Priscila Missaki Nakamura $^{2} \cdot$ Camila Bosquiero Papini $^{3}$
}

Received: 13 January 2021 / Accepted: 10 July 2021 / Published online: 5 August 2021

(c) The Author(s), under exclusive licence to Springer-Verlag Italia S.r.l., part of Springer Nature 2021

\begin{abstract}
Purpose COVID-19 pandemic has disrupted the lives of people of all nations. This study examined physical activity (PA) behavior, barriers and facilitators to PA during the COVID-19 pandemic.

Methods This cross-sectional study assessed adults using a survey comprised of two parts: (a) demographics and general health and; (b) PA-related questions. The survey link was disseminated via email and various social media, and was active from September to October 2020.

Results A total of 277 adults (Mean $\pm \mathrm{SD}$; age $=32.6 \pm 13.6$ years, $\mathrm{BMI}=27.1 \pm 16.5 \mathrm{~kg} / \mathrm{m}^{2}$ ) were evaluated. A majority of the sample was female (67\%), single (53\%) and White (70\%). About a third of the participants reported good mental and physical health, with a similar amount reporting weight gain during the pandemic. Participants further reported on average $271 \mathrm{~min}$ of PA/week, and $5.7 \mathrm{~h} /$ day of sitting time. Overall, $41.5 \%$ of the participants reported a decrease in PA during the COVID-19 pandemic, but those not meeting PA recommendations reported higher rates (67.9\%) of decrease in levels of PA than their active counterparts $(23.6 \%) ; x^{2}(2, N=277)=55.757, p<0.01$. Over $50 \%$ of the participants reported engaging in PA at home, with significantly more females (43.5\%) than males (17.6\%) making use of live stream PA/exercise session opportunities; $x^{2}(2, N=277)=18.896, p<0.001$. "Closed gyms" and "more time" were reported as the main negative and positive factors, respectively, affecting PA during the pandemic.

Conclusions Our findings suggest that PA behavior was negatively affected in US adults during the COVID-19 pandemic, and that "closed gym" (i.e., barrier), and "more time" (i.e., facilitator), were the main factors reported affecting PA participation. Given the well-known public health importance of PA, it is paramount that public health initiatives focus on providing not only educational but also environmental opportunities and support for PA during this period.
\end{abstract}

Keywords Physical activity $\cdot$ Coronavirus $\cdot$ Healthy lifestyle $\cdot$ Sitting time

\section{Introduction}

By the end of December 2019, China reported to the World Health Organization (WHO) an outbreak of pneumonia of unknown etiology in the Province of Hubei, a landlocked province in Central China with nearly 59 million inhabitants.

Emerson Sebastião

esebastiao@niu.edu

$1 \quad$ Health and Exercise Research Group, Department of Kinesiology and Physical Education, Northern Illinois University, 1425 Lincoln Highway, DeKalb, IL 60115, USA

2 Federal Institute of Education, Science and Technology, Muzambinho, Minas Gerais, Brazil

3 Department of Sport Sciences, Federal University of Triângulo Mineiro, Uberaba, Minas Gerais, Brazil
Later, Chinese authorities identified a novel coronavirus (i.e., SARS-CoV-2), and on March 11, 2020, the WHO declared the novel coronavirus outbreak-associated disease COVID-19-a global pandemic after cases of the virus increased 13-fold in the two weeks prior [1].

The COVID-19 pandemic has drastically disrupted the daily lives of people throughout the globe. Following recommendations from public health specialists, government leaders established measures to contain the rapid spread of the virus. In the United States, social distancing and shelter-inplace orders, along with closures of non-essential business and activity spaces such as recreational centers and gyms were put into place to mitigate the spread of the COVID19. Although important, such strategies may have negative consequences on health behaviors such as physical activity (PA) and related health outcomes [2-4]. For instance, Fitbit 
global data showed significant declines in the amount of steps (an objective measure of PA) in different parts of the globe. Decreases in steps were noticed in at least 26 countries with the largest decrease in Spain, 38\%. In the US, the decrease was about $12 \%$ [5]. In addition, there is some evidence that COVID-19 social distancing measures may have also changed the perceptions of barriers and facilitators to PA [6].

A physically active lifestyle is associated with a large array of physical $[7,8]$ and psychological benefits $[9,10]$, including but not limited to chronic disease prevention and treatment, and improved well-being $[8,11]$. By contrast, a sedentary/physically inactive lifestyle has been associated with poor physical and mental health, as well as premature death [12-16].

Like European countries, US faced different "waves" of the virus, and a global concern about the impact COVID-19 physical distancing measures installed around PA participation. In fact, few recent studies conducted during the "first wave" have demonstrated that the COVID-19 pandemic has negatively affected PA participation [2, 17]. For example, a study conducted by a Canadian group demonstrated that among individuals considered inactive, approximately $40 \%$ became less active, and among their active participants $22.4 \%$ reported reductions in PA during the pandemic [2].

Building upon the limited evidence on the impact of COVID-19 social distancing measures on activity behavior, the purpose of the present study was to examine PA, barriers and facilitators to PA during the COVID-19 pandemic among US adults. We hypothesized that US adults would report low levels of PA and higher amounts of sitting time during this period, with a significant proportion of the participants reporting decreases in PA during this period along with weight gains.

\section{Methods}

\section{Study design}

This cross-sectional survey study was approved by the Institution Review Board of Northern Illinois University (IRB; protocol \# HS21-0071) in compliance with the Human Subjects Guidelines, and electronic consent was acquired from all participants prior to data collection. Google forms, which is a survey administration software that is included in the Google Docs Editors software suite was used to develop the survey [18], that later was electronically distributed via various social media (e.g., Facebook, Instagram, Twitter and WhatsApp messenger), to primarily assess PA perceptions during the COVID-19 pandemic among US adults. Google Docs Editors software allows the collection of information of interest from a variety of populations and the collected information can be automatically entered into a spreadsheet [18].

\section{Participants}

A total of 301 individuals aged 18 years and older responded to the survey. Inclusion criteria included: (a) aged 18 years and over and, (b) live in the US. A research staff supervised by the principal investigator entered and checked the survey data on Excel before transferring to SPSS. Duplicated answers were observed for 24 participants and therefore, the second entry was excluded from the analysis. Thus, the final analytical sample was comprised of 277 participants $(67.1 \%$ females). The vast majority of the respondents were from the State of Illinois; however, individuals from other 22 states were represented.

\section{Survey}

The electronic survey was developed using Google forms [18] and comprised of two parts. The entire survey could be completed within 7-10 min. The first part contained 14 questions related to basic demographics (e.g., age, sex, level of education, marital status, race) and general health information (e.g., perceived mental and physical health, presence of chronic diseases, perceptions of weight gain during the COVID-19 pandemic). The second part contained 8 questions related to PA, sitting time, in addition to barriers and facilitators to PA. These questions aimed to identify PA level and perceptions about changes in PA level during the COVID-19 pandemic, sitting time, as well as main barriers and facilitators to PA. Specific PA and sitting questions included: (a) "Since the start of the COVID-19 US pandemic and the social distancing/shelter-in-place order, would you say your engagement in physical activity/exercise has: increased/ decreased/ stayed the same"; (b) "On average, how many days per week did you engage in physical activity/exercise since the start of the coronavirus pandemic and social distancing/shelter-in-place orders? Open-ended questions-number of days"; (c) "On average, how much time did you usually spend on those days doing physical activity/ exercise? Open-ended questions-time"; (d) "During the lockdown period of non-essential activities (with gyms and recreational centers closed), where did you mostly engaged in physical activity/exercise? At home/ at the park/ other outdoor area/ I did not engage in physical activity/exercise during the lockdown"; (e) "During the lockdown period, did you participate in any live physical activity/exercise classes through social media (YouTube, Instagram, Facebook, etc.)? YES/NO" and; (f) "On average, how much time you usually spend sitting on a weekday since the start of the coronavirus pandemic and social distancing/shelter-in-place-orders? Open-ended-time". A copy of the entire survey questions/ 
questionnaire as well as the data collected are available by the principal investigator (ES) upon request. Of note, the questionnaire was specifically developed to address the purpose of the present study. Although the psychometric properties of the questionnaire were not tested, we pilot-tested it in small groups of individuals that did not participate in the study. Further, the questionnaire was also evaluated by two researchers with experience in online surveys. The feedback gathered from the small group of individuals and the two researchers were used to revise/improve the questionnaire before it was broadly disseminated.

The survey link was first distributed via email to all faculty in the Department of Kinesiology and Physical Education (KNPE) explaining the study's purpose and asking them to forward the survey link to all their students. Faculty were also instructed to ask students to disseminate the link of the survey onto their social media accounts (e.g., Facebook, Instagram, and Twitter). The principal investigator further sent the same email to three other Northern Illinois University faculty outside the KNPE department (i.e., Department of Education Technology, and Department of Health Studies) with the same instructions. The survey was active from September to October 2020. During this period, the State of Illinois (origin of the survey) was in phase 3; however, with reopening differences in different regions within Illinois.

\section{Data analysis}

All analyses were conducted using SPSS version 26 (IBM Corporation, Armonk, NY, USA). Descriptive statistics were used for sample characterization. Pearson correlation was used to examine the association between PA and sitting time. Chi-square test was used to compare outcomes for select closed-ended questions, and independent $t$ test and one-way ANOVAs with Bonferroni correction were used to compare continuous PA and sitting time data among selected variables. Barriers and facilitators for PA were analyzed using frequency analysis. Significance level was set at $p<0.05$ for all pertinent analyses.

\section{Results}

\section{Demographics and health}

Detailed demographic and health information about the sample is displayed in Table 1. Briefly, participants responding to the survey presented with an average age of approximately 33 years with the majority of the participants being female, self-reported as White, and on average were classified as overweight based on body mass index values. About one third of the participants further classified their physical and
Table 1 General demographic and health characteristics of 277 US adult individuals responding to the short electronic survey on physical activity during the COVID-19 pandemic between September and October 2020

Overall $(n=277)$

Demographics

Age, years

$32.6(13.6)$

Sex, \% of female

67.1

Marital status, $\%$

Single

Married

40.1

Divorced

6.1

Widowed

1.4

Other

Race, \%

White

African American

8.3

Latinx

15.2

Asian

2.2

Multiple races

4.3

Education, \%

High school/GED $\quad 5.8$

Some college $\quad 27.1$

Bachelors or more $\quad 49.4$

Health

BMI, $\mathrm{kg} / \mathrm{m}^{2}$ (SD)

$27.1(16.5)$

Physical health, \%

Excellent

19.1

Very good

26.7

Good

35.0

Fair

13.7

Poor

5.4

Mental health, \%

Excellent

6.5

Very good

19.1

Good

34.7

Fair

26.0

Poor

Chronic diseases, $\%$ yes

Type 2 diabetes

0.4

High blood pressure

5.4

Cancer

2.9

Arthritis

7.9

Asthma

10.8

Stroke

Weight during COVID-19, \%

Gained

33.2

Lost

25.6

Stayed the same

41.2

$G E D$ general education development, $B M I$ body mass index, ${ }^{£} p$ for independent $t ;{ }^{¥} p$ for Chi-square 
mental health as good, with a similar rate reporting weight gain during the COVID-19 pandemic.

\section{Self-reported physical activity and sedentary behavior}

On average, participants reported 271.17 (252.4) minutes per week of PA and 5.7 (3.3) hours per day of sitting time, with Pearson $r$ correlation revealing a significant small negative association between PA and sitting time $(r=-0.22$; $p<0.001)$. No significant differences were observed for PA [females: 259.7 (240.6) vs. males: 294.7 (274.7) minutes per week; $t(275)=-1.084 ; p=0.279$ ] or sitting time [females: 5.7 (3.3) vs. males: 5.7 (3.3) hours per day; $t(275)=0.041$; $p=0.967]$ between sex. In addition, $59 \%$ of the participants meet the current World Health Organization recommendations for PA (i.e., 150-300 min per week of moderate-tovigorous $\mathrm{PA}$ ), with more males (63.5\%) compared to females $(57.5 \%)$ meeting the recommendations; however, such difference was not significant $\left(x^{2}(1, N=277)=0.978, p=323\right)$.

Additional analysis focused on potential differences in self-reported PA and sitting time during the COVID-19 pandemic according to the overall perception of physical and mental health status, and perception of weight change during the pandemic revealed significant differences among groups. Briefly, one-way ANOVA revealed significant differences in PA and sitting time among groups with a different perception of overall physical health and significant differences in PA among groups of different overall perception of mental health (Table 2).

Follow-up Bonferroni post hoc test revealed that individuals reporting their physical health as excellent/very good reported significantly higher levels of PA per week compared to those reporting their physical health as good $(p<0.001)$ or fair/poor $(p<0.001)$. No other significant differences were observed among physical health groups regarding PA. In terms of sitting time, the post hoc analysis revealed that individuals reporting their physical health as excellent/very good reported significantly less amount of sitting time than those reporting their physical health as good $(p=0.007)$ or fair/poor $(p<0.001)$. In addition, a significant difference was also observed for sitting time between those reporting good and fair/poor physical health $(p=0.019)$.

In terms of mental health, Bonferroni analysis revealed that individuals reporting their mental health as fair/poor reported significantly higher amounts of sitting time than those reporting their mental health as good $(p=0.013)$. No other significant differences were observed among mental health groups regarding sitting time.

One-way ANOVA further revealed significant differences in PA among groups with a different perception of weight change but no differences on sitting time (Table 3). Follow-up post hoc analysis revealed that individuals reporting weight gain during the pandemic reported significantly less PA participation per week compared to those reporting weight loss $(p<0.001)$ or no changes in weight $(p=0.002)$. In addition, a significant difference in PA participation was observed between those reporting weight loss and those with no changes in weight (stayed the same; $p=0.034$ ).

\section{Perceptions of physical activity during the COVID-19 pandemic}

Figure 1 displays detailed information on the perception of changes in PA during the COVID-19 pandemic overall and separated by sex, groups of different overall perception of physical and mental health, and weight change. Overall, the results show that less than a third of the participants reported an increase in PA and $41.5 \%$ reported a decrease in PA during this period. The Chi-square test did

Table 2 Time spent in physical activity and sitting time separated by physical and mental health status during the COVID-19 pandemic of US adults, and results of the analysis of variance

\begin{tabular}{|c|c|c|c|c|c|c|}
\hline & \multirow[t]{2}{*}{ Overall $(n=277)$} & \multicolumn{3}{|l|}{ Physical health } & \multirow[t]{2}{*}{$F$} & \multirow[t]{2}{*}{$P$} \\
\hline & & $\begin{array}{l}\text { Excellent/very good } \\
(n=127)\end{array}$ & Good $(n=97)$ & Fair/poor $(n=53)$ & & \\
\hline Total PA, min.week & $271.2(252.4)$ & $394.0(242.0)$ & $196.9(222.0)$ & $112.6(179.7)$ & 37.600 & $<0.001$ \\
\hline \multirow[t]{3}{*}{ Sitting time, h.day } & $5.7(3.3)$ & $4.7(2.5)$ & $6.0(3.0)$ & $7.5(4.4)$ & 15.429 & $<0.001$ \\
\hline & \multirow[t]{2}{*}{ Overall $(n=276)$} & \multicolumn{3}{|l|}{ Mental health $^{\neq}$} & \multirow[t]{2}{*}{$F$} & \multirow[t]{2}{*}{$P$} \\
\hline & & $\begin{array}{l}\text { Excellent/very good } \\
(n=71)\end{array}$ & Good $(n=96)$ & Fair/poor $(n=109)$ & & \\
\hline Total PA, min.week & $268.9(249.9)$ & 299.3 (209.9) & $293.6(262.3)$ & $227.2(259.0)$ & 2.539 & 0.081 \\
\hline Sitting time, h.day & $5.7(3.3)$ & $5.6(3.5)$ & $5.1(3.0)$ & $6.4(3.3)$ & 4.207 & 0.016 \\
\hline
\end{tabular}

$P A$ physical activity, min.week minutes per week, h.day hours per day

${ }^{\ddagger}$ Missing data; $n=1$ 
Table 3 Time spent in physical activity and sitting time separated by perception of weight change during the COVID-19 pandemic of US adults, and results of the analysis of variance

\begin{tabular}{lccccr}
\hline & Overall $(n=277)$ & \multicolumn{2}{l}{ Weight perception } & & $F$ \\
\cline { 3 - 6 } & & Gained $(n=92)$ & Lost $(n=71)$ & Stayed the same $(n=114)$ & $P$ \\
\hline Total PA, min.week & $271.2(252.4)$ & $169.1(239.9)$ & $378.9(257.5)$ & $286.5(228.3)$ & 15.725 \\
Sitting Time, h.day & $5.7(3.3)$ & $6.0(3.5)$ & $5.1(2.8)$ & $5.9(3.3)$ & $<0.001$ \\
\hline
\end{tabular}

$P A$ physical activity, min.week minutes per week, h.day hours per day

${ }^{\ddagger}$ Missing data; $n=1$
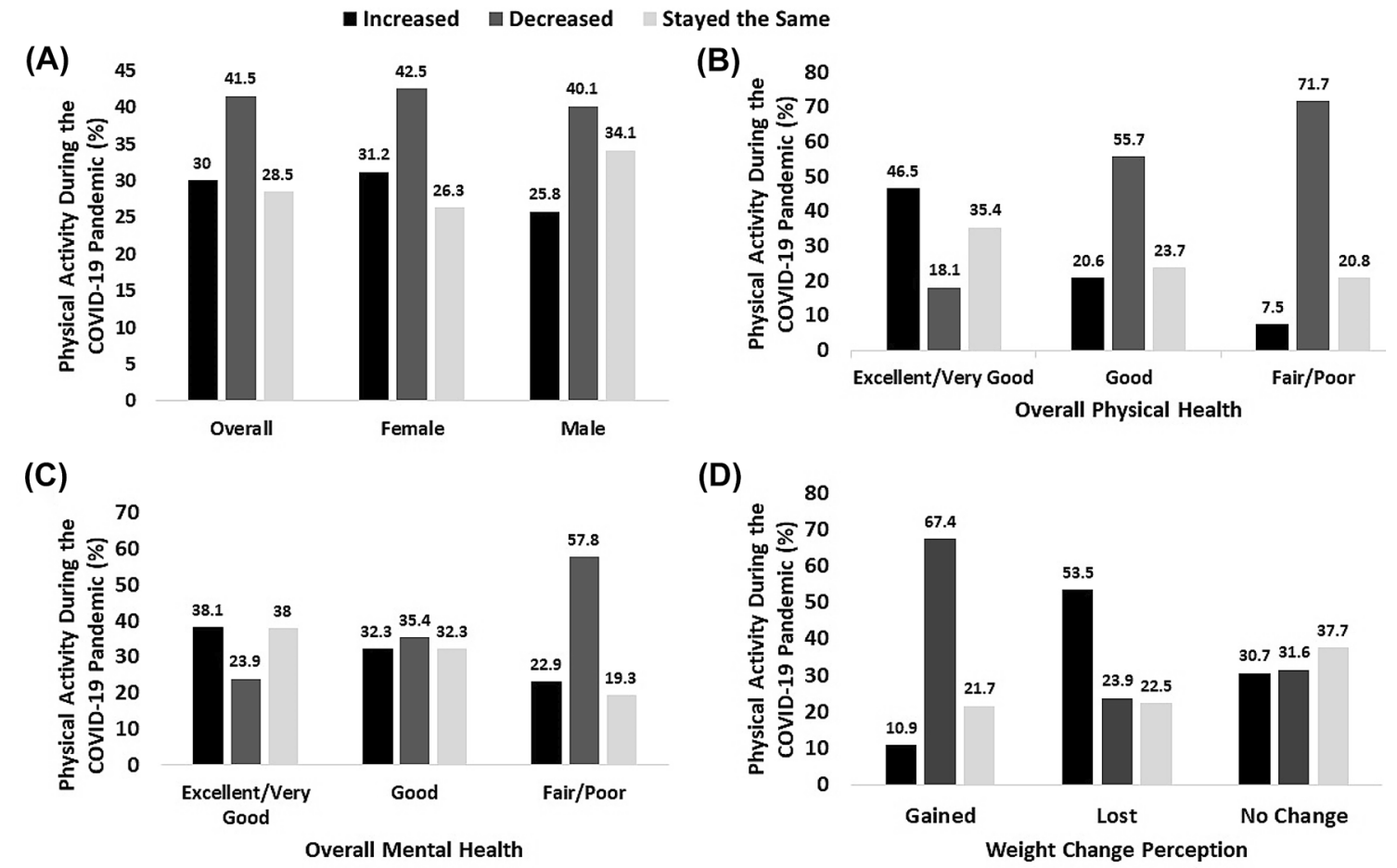

(D)

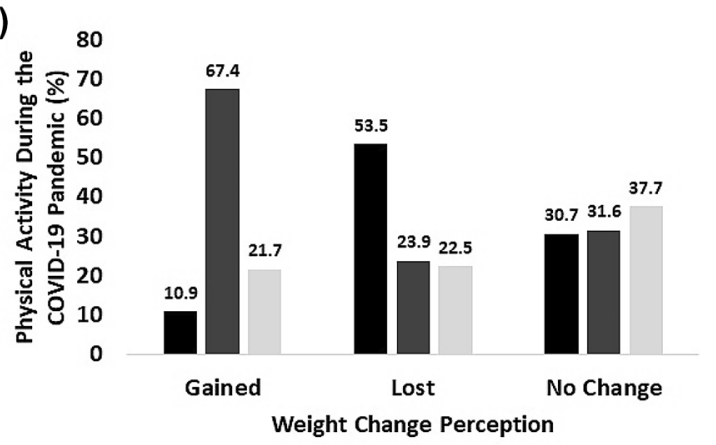

Fig. 1 Perception of changes in physical activity during the COVID-19 pandemic of 277 US adults overall and separated by sex (A), physical health $(\mathbf{B})$, mental health $(\mathbf{C})$, and weight change (D)

not reveal significant differences in the proportion of males and females in terms of perception of change in PA during the COVID-19 pandemic (i.e., increased vs. decreased vs. stayed the same) $\left[x^{2}(2, N=277)=1.345, p=0.510\right]$, but did point out significant differences for groups of different physical $\left[x^{2}(4, N=277)=60.348, p<0.001\right]$ and mental $\left[x^{2}(8\right.$, $N=277)=37.724, p<0.001]$ health, and weight change $\left[x^{2}\right.$ $(4, N=277)=22.643, p<0.001]$.

Chi-square test revealed significant differences $\left[x^{2}(2\right.$, $N=277)=55.757, p<0.01]$ in the proportion of individuals meeting and not meeting current PA recommendations in terms of the perception of change in PA during the COVID-19 pandemic. Among those not meeting current PA recommendations ( $40 \% ; n=112), 12.5 \%$ reported an increase in PA during the pandemic, while $67.9 \%$ reported a decrease and $19.6 \%$ reported that their
PA level stayed the same. On the other hand, of individuals meeting PA recommendations $(\sim 60 \% ; n=165), 41.8 \%$ reported increases in PA, while $23.6 \%$ reported decreases and $34.5 \%$ reported that their PA level stayed the same.

Results of the survey further revealed that the preferred place to partake in PA during the COVID-19 pandemic period was reported to be "at home" $(52 \%)$, followed by "other outdoor area" (33.6\%). A small percent $(6.1 \%)$ of participants reported the use of "parks" to be physically active during this period. No significant difference was observed between sex in terms of PA preferred places $\left[x^{2}(3, N=277)=2.083, p=0.555\right]$. In addition, $35 \%$ of the participants reported participation in live stream PA/exercise sessions via social media during the pandemic; with significantly more females (43.5\%) partaking 
in such opportunity compared to males $(17.6 \%)\left[x^{2}(2\right.$, $N=277)=18.896, p<0.001]$.

\section{Barriers and facilitators to physical activity during the COVID-19}

Table 4 displays in detail the main barriers and facilitators reported by the 277 surveyed participants. Frequency analysis demonstrated that "closed gym" was the most frequently reported barrier whist "more time" was the most frequently reported facilitator to PA.

\section{Discussion}

This study examined activity behavior during the COVID19 pandemic in US adults using a short online survey. More specifically, we examined the extent to which the quarantine (social isolation and shelter-in-place order) affected aspects of PA and sedentary behavior in US adults. The main findings were (a) PA was not different between males and females, but was different when compared among groups with different perceptions of physical and mental health, and weight change during the pandemic; (b) sitting time was not different between sex, but significantly different among groups with different perceptions of physical health, and weight change; (c) the majority of the participants met current recommendations for PA; (d) nearly $41 \%$ of the participants reported a decrease in PA during the COVID-19 pandemic with large proportions among those reporting overall physical and mental health as fair/poor or weight gain during the pandemic; (e) the majority of the participants reported engaging in PA at home, with significantly more females than males making use of live stream PA/exercise session opportunities; and (f) closed gyms and more time were reported as the main negative and positive factors, respectively, affecting PA during the pandemic. Our

Table 4 Main barriers and facilitators to physical activity during COVID-19 pandemic reported by 277 US adult individuals between September and October of 2020

\begin{tabular}{lllll}
\hline Barriers & Frequency & Facilitators & Frequency \\
\hline 1 Closed gym & 100 times & 1 & More time & 98 times \\
2 Lack of motivation & 68 times & 2 & $\begin{array}{c}\text { Ability to } \\
\text { exercise at } \\
\text { home }\end{array}$ & 62 times \\
3 Work schedule & 41 times & 3 & $\begin{array}{c}\text { Having a } \\
\text { buddy to } \\
\text { be active } \\
\text { outdoors }\end{array}$ & 26 times \\
$4 \begin{array}{c}\text { Lack of equipment at } \\
\text { home }\end{array}$ & 34 times & & - & - \\
\hline
\end{tabular}

findings, partially corroborate our hypothesis on PA and sitting time in US adults during the COVID-19 pandemic.

The COVID-19 pandemic has greatly affected people's lives worldwide, including disrupting health behaviors. Although the participants of the present study reported, on average, PA values during the COVID-19 pandemic that classify them as active for health benefits ( $\sim 60 \%$ of the participants achieved current PA recommendations), the average amount of sitting time (a proxy of sedentary behavior) reported by the participants can be viewed as high. Variations in PA and sitting time became more evident when data were analyzed according to groups of different perceptions of physical and mental health, and weight change. Those reporting their physical and mental health as fair/poor and/or reporting weight gains during the pandemic reported general lower levels of PA and high levels of sedentary behavior (i.e., sitting time) than their counterparts. These findings should be a concern as there is evidence suggesting sedentary behavior as an important and independent health risk behavior [19-21]. For example, researchers have suggested that high amounts of sedentary behavior (e.g., sitting time) is an independent health risk factor for cardiovascular diseases and other conditions even for those meeting PA guidelines [22, 23]. Although there are some general qualitative guidelines on sedentary behavior, Stamatakis et al. [24] recently noted that public health lacks consistent evidence on this matter, suggesting that the development of quantitative guidelines on sedentary behavior using underdeveloped evidence base is premature.

We observed that overall, nearly $41 \%$ of our participants reported a decrease in PA during the COVID-19 pandemic. Follow-up analysis looking at groups of physical and mental health, and perception of weight change demonstrated that the number of individuals reporting decreases in PA was higher in those reporting their physical and mental health as fair/poor, reporting weight gains, or not meeting current PA guidelines. Our findings corroborate the results of a recent study conducted among diseased population and recent preliminary results from an online survey collecting information on PA and dietary behaviors in eight languages before and during home confinement due to COVID-19 [2, 17, 25]. The latter demonstrated a significant decrease in PA during the pandemic throughout the PA intensity continuum (i.e., vigorous and moderate intensity: 33\% and; walking: 34\%). In addition, a group of researchers [2] investigating how social distancing and closure of city and provincial recreation facilities, national parks and playgrounds impacted PA behavior and well-being among Canadians observed that $40.5 \%$ of their inactive participants became less active, compared to only $22.4 \%$ of the active participants becoming less active. The researchers further observed that one third of the inactive participants became more active, and nearly $40 \%$ of the active individuals became more active. Collectively, the 
findings of the present and recently published studies show that although necessary, social distancing and shelter-inplace measures, along with the closure of non-essential business (e.g., gyms, recreation centers) seemed to negatively affect PA behavior in adult individuals. This is a concern due to the detrimental impact on the health of a physically inactive/sedentary lifestyle [26-28]. Lesser and Nienhuis observed significant differences in well-being among inactive individuals who reported an increase, decrease or no changes in PA during the COVID-19 pandemic, with worst well-being scores being observed for those reporting reductions in PA [2]. By contrast, PA has shown to decrease the chances of experiencing a severe case of COVID-19. A study using a Mendelian approach observed that PA was associated with fivefold decreased risk of severe COVID19 , but not with hospitalization, while body mass index and lifetime smoking were found to increase the risk of severe COVID-19 and hospitalization by twofold [29]. These findings highlight the importance of maintaining a healthy lifestyle to protect against severe COVID-19 symptoms (upon contracting the virus) and underscores the public health value of PA in the fight against the coronavirus pandemic.

Fifty-two percent of our participants reported being physically active at home, while $33.6 \%$ reported to be active in "other outdoor area" and only a small portion $6.1 \%$ reported the use of parks to be physically active during the COVID19 pandemic. In addition, 35\% of our participants reported participation in live stream PA/exercise sessions via social media. These findings partially reflect the social distancing measures and the closure of non-essential business (e.g., gyms and recreational centers), where individuals reduced the frequency of outdoor activities [30], but may understand the need to engage in healthy behaviors at home to maintain health. One study examined the influencing mechanism of health consciousness on home-based exercise during the COVID-19 pandemic in a sample of 449 Chinese individuals. The authors observed that health consciousness had a positive effect on home-based exercise, and according to the authors, perceived behavioral control (an individual's estimation of how difficult it is to perform a given behavior [31]) served as a mediator between health consciousness and home-based exercise. To this end, developing and implementing ways to improve one's perception of control may be an important step towards keeping individuals active at home during confinement, and engender positive health outcomes [32].

We reported that $41.5 \%$ of the participants in this study reported reductions in PA during the pandemic, while $30 \%$ reported an increase. These findings can be partially explained by perceived barriers and facilitators to PA during this period. In our sample, "closed gym", "lack of motivation", "work schedule" and "lack of equipment at home" were reported as main barriers to engage in PA, while "more time", "ability to exercise at home", "having a buddy to be active outdoors" as facilitators. At the time of writing, we did not find research studies investigating barriers and facilitators to PA in adults. However, our findings are somewhat similar to those reported in a study conducted with 1214 Irish adolescents (12-18 years old) where the main barriers were "coronavirus" and "club training cancelled" while facilitators were "more time" and "no school" [6] but such comparison is limited due to the age difference between participants. On a broad view, it is possible to observe that the COVID-19 pandemic slightly changed the perception/composition of factors affecting PA. Before COVID-19, one of the most frequently reported barriers to PA was lack of time $[33,34]$, which during the pandemic emerged as facilitator as "more time". In addition, the report of "lack of equipment at home" as barrier to PA by a significant portion of our participants shows that despite efforts to promote PA in its large array of possibilities, a more infusive strategy needs to be put in place to clarify that PA and health benefits can be achieved without specific equipment.

\section{Limitations}

Our study has some limitations. The survey was active during the months of September and October. Thus, we do not know whether similar results would be obtained if the study was conducted earlier in the confinement. Although we have responses from 23 different states, the majority of our sample was from the State of Illinois. This is an important point because during the survey period, each state was at different phases of their reopening plans and had divergent mitigation practices. Also, we did not perform a sample size calculation. However, it is believed that a minimum sample of 100 participants are capable of engendering meaningful/accurate results. In addition, all information collected in terms of PA and sitting time were self-reported, therefore, with the possibility of overestimation and underestimation. Also, information on physical and mental health and weight change perception were collected using single questions, which can also be viewed as a limitation. Despite the limitations, we were able to contribute valuable information regarding PA and sedentary behavior during the COVID-19 pandemic among US adults during the months of September and October, where US experienced the "second wave".

\section{Conclusions}

We presented results of a short survey conducted during the "second wave" of the COVID-19 pandemic suggesting that PA behavior was negatively affected in US adults during this period. We have shown that PA participation and sitting time did not differ between males and females, but significant 
differences were observed for groups of individuals with different perceptions of physical and mental health, with those classifying their overall physical and mental health as excellent/very good presenting with higher levels of PA and lower amounts of sitting time than their counterparts perceiving their physical and mental health as good or fair/poor. We further observed higher amounts of PA for those reporting weight loss during the COVID-19 pandemic. In addition, the results showed that a large number of individuals perceived a decrease in PA during this period, especially among those classifying their physical and mental health as fair/poor and/ or reporting weight gain during this period. Our findings also showed that the preferred place to partake in PA was at home and that more females than males took advantage of live stream PA/exercise classes via social media. Closed gyms and lack of motivation were the main barriers to PA reported by the participants, while more time and ability to exercise at home emerged as the main facilitators.

Currently, the vaccine/immunization is being administrated at an arguably fast pace in different parts of the globe, including in the US - where nearly 171 million individuals (52.2\%) received at least 1 dose of the vaccine and about 140 million (42.6) have been fully vaccinated (as on June 6, 2021). However, the COVID-19 still poses challenges related to healthy lifestyle behaviors including PA and consequent health outcomes. To this end, it is imperative to advocate and provide not only educational, but also environmental opportunities and support for PA during this period, as phased re-openings continue and new closures may possibly occur. This is important not only because of the increased prevalence of mental illness due to confinement, but also because being physically active can drastically reduce the chances of severe COVID-19 symptoms if infected.

Author contributions ES, MS, PMN and CBP: study concept and design, survey development, data analysis, manuscript drafts and final version.

Funding The authors received no financial support with respect to the research, authorship, and/or publication of this article.

Availability of data and materials Survey questions and data collected will be made available by the first author upon request.

Code availability Not applicable.

\section{Declarations}

Conflict of interest The authors declare no conflict of interest related to this work.

Ethics approval The Northern Illinois University Institution Review Board approved the present study protocol (protocol number HS210071).

\section{References}

1. Director-general, W. H. O (2020) WHO Director-General's opening remarks at the Mission briefing on COVID-19. https://www. Who.Int/Dg/Speeches/Detail/Who-Director-General-S-OpeningRemarks-At-the-Media-Briefing-on-Covid-19---11-March-2020

2. Lesser IA, Nienhuis CP (2020) The impact of COVID-19 on physical activity behavior and well-being of Canadians. Int J Environ Res Public Health. https://doi.org/10.3390/ijerph17113899

3. Hemphill NM, Kuan MTY, Harris KC (2020) Reduced physical activity during COVID-19 pandemic in children with congenital heart disease. Can J Cardiol. https://doi.org/10.1016/j.cjca.2020. 04.038

4. Meyer J, Herring M, McDowell C, Lansing J, Brower C, Schuch $F$ et al (2020) Joint prevalence of physical activity and sitting time during COVID-19 among US adults in April 2020. Preventive Med Rep 20:101256. https://doi.org/10.1016/j.pmedr.2020. 101256

5. Fitbit (2020) The impact of coronavirus on global activity. Fitbit blog

6. Ng K, Cooper J, McHale F, Clifford J, Woods C (2020) Barriers and facilitators to changes in adolescent physical activity during COVID-19. BMJ Open Sport Exerc Med. https://doi.org/10.1136/ bmjsem-2020-000919

7. Bull F, Saad Al-Ansari S, Biddle S, Borodulin K, Buman M, Cardon $\mathrm{G}$ et al (2020) World Health Organization 2020 guidelines on physical activity and sedentary behaviour. Br J Sports Med. https://doi.org/10.1136/bjsports-2020-102955

8. Pedersen BK, Saltin B (2015) Exercise as medicine - Evidence for prescribing exercise as therapy in 26 different chronic diseases. Scand J Med Sci Sports. https://doi.org/10.1111/sms.12581

9. Chekroud SR, Gueorguieva R, Zheutlin AB, Paulus M, Krumholz HM, Krystal JH, Chekroud AM (2018) Association between physical exercise and mental health in 1.2 million individuals in the USA between 2011 and 2015: a cross-sectional study. Lancet Psychiatry. https://doi.org/10.1016/S2215-0366(18)30227-X

10. Stubbs B, Vancampfort D, Hallgren M, Firth J, Veronese N, Solmi $M$ et al (2018) EPA guidance on physical activity as a treatment for severe mental illness: a meta-review of the evidence and position statement from the European Psychiatric Association (EPA), supported by the international organization of physical therapists in mental. Eur Psychiatry. https://doi.org/10.1016/j.eurpsy.2018. 07.004

11. Marquez DX, Aguinãga S, Vásquez PM, Conroy DE, Erickson KI, Hillman C et al (2020) A systematic review of physical activity and quality of life and well-being. Trans Behav Med 10(5):10981109. https://doi.org/10.1093/tbm/ibz198

12. Vancampfort D, Hallgren M, Schuch F, Stubbs B, Smith L, Rosenbaum $S$ et al (2020) Sedentary behavior and depression among community-dwelling adults aged $\geq 50$ years: results from the Irish longitudinal study on Ageing. J Affect Disord. https://doi.org/10. 1016/j.jad.2019.11.066

13. Ashdown-Franks G, Koyanagi A, Vancampfort D, Smith L, Firth J, Schuch F et al (2018) Sedentary behavior and perceived stress among adults aged $\geq 50$ years in six low- and middle-income countries. Maturitas. https://doi.org/10.1016/j.maturitas.2018. 08.005

14. Stubbs B, Vancampfort D, Firth J, Schuch FB, Hallgren M, Smith L et al (2018) Relationship between sedentary behavior and depression: a mediation analysis of influential factors across the lifespan among 42,469 people in low- and middle-income countries. J Affect Disord. https://doi.org/10.1016/j.jad.2017.12.104

15. Kokkinos P, Sheriff H, Kheirbek R (2011) Physical inactivity and mortality risk. Cardiol Res Pract. https://doi.org/10.4061/2011/ 924945 
16. Booth FW, Roberts CK, Laye MJ (2012) Lack of exercise is a major cause of chronic diseases. Compr Physiol. https://doi.org/ 10.1002/cphy.c110025

17. Ammar A, Brach M, Trabelsi K, Chtourou H, Boukhris O, Masmoudi L et al (2020) Effects of COVID-19 home confinement on eating behaviour and physical activity: results of the ECLBCOVID19 international online survey. Nutrients. https://doi.org/ $10.3390 /$ nu 12061583

18. Narayanaswamy Vasantha Raju, Harinarayana NS (2016) Online survey tools: a case study of Google Forms Online. National Conference on Scientific, Computational \& Information Research Trends in Engineering, GSSS-IETW, Mysore

19. Thorp AA, Owen N, Neuhaus M, Dunstan DW (2011) Sedentary behaviors and subsequent health outcomes in adults: a systematic review of longitudinal studies, 19962011. Am J Prev Med 41(2):207-215. https://doi.org/10.1016/j.amepre.2011.05.004

20. Dunstan DW, Barr ELM, Healy GN, Salmon J, Shaw JE, Balkau B et al (2010) Television viewing time and mortality: the Australian diabetes, obesity and lifestyle study (ausdiab). Circulation 121(3):384-391. https://doi.org/10.1161/CIRCULATIONAHA. 109.894824

21. Owen N, Sparling PB, Healy GN, Dunstan DW, Matthews CE (2010) Sedentary behavior : emerging evidence for a new health risk. Mayo Clin Proc 85(12):1138-1141. https://doi.org/10.4065/ mcp.2010.0444

22. Chomistek AK, Manson JE, Stefanick ML, Lu B, Sands-Lincoln M, Going SB et al (2013) Relationship of sedentary behavior and physical activity to incident cardiovascular disease: results from the women's health initiative. J Am Coll Cardiol. https://doi.org/ 10.1016/j.jacc.2013.03.031

23. Lavie CJ, Ozemek C, Carbone S, Katzmarzyk PT, Blair SN (2019) Sedentary behavior, exercise, and cardiovascular health. Circ Res. https://doi.org/10.1161/CIRCRESAHA.118.312669

24. Stamatakis E, Ekelund U, Ding D, Hamer M, Bauman AE, Lee IM (2019) Is the time right for quantitative public health guidelines on sitting? A narrative review of sedentary behaviour research paradigms and findings. Br J Sports Med. https://doi.org/10.1136/ bjsports-2018-099131

25. Sassone B, Mandini S, Grazzi G, Mazzoni G, Myers J, Pasanisi G (2020) Impact of COVID-19 pandemic on physical activity in patients with implantable cardioverter-defibrillators. J Cardiopulm Rehabil Prev. https://doi.org/10.1097/HCR.0000000000000539
26. Marçal IR, Fernandes B, Viana AA, Ciolac EG (2020) The urgent need for recommending physical activity for the management of diabetes during and beyond COVID-19 outbreak. Front Endocrinol. https://doi.org/10.3389/fendo.2020.584642

27. De Rezende LFM, Lopes MR, Rey-Loṕez JP, Matsudo VKR, Luiz ODC (2014) Sedentary behavior and health outcomes: an overview of systematic reviews. PLoS ONE. https://doi.org/10.1371/ journal.pone. 0105620

28. Lee I-M, Shiroma EJ, Lobelo F, Puska P, Blair SN, Katzmarzyk PT (2012) Impact of physical inactivity on the world's major noncommunicable diseases. Lancet 380(9838):219-229. https://doi. org/10.1016/S0140-6736(12)61031-9.Impact

29. Li S (2020) Modifiable lifestyle factors and severe COVID-19 risk: evidence from Mendelian randomization analysis. medRxiv. https://doi.org/10.1101/2020.10.19.20215525

30. Coroiu A, Moran C, Campbell T, Geller AC (2020) Barriers and facilitators of adherence to social distancing recommendations during COVID- 19 among a large international sample of adults. PLoS ONE. https://doi.org/10.1371/journal.pone.0239795

31. Ajzen I, Madden TJ (1986) Prediction of goal-directed behavior: attitudes, intentions, and perceived behavioral control. J Exp Soc Psychol. https://doi.org/10.1016/0022-1031(86)90045-4

32. Solon Júnior LJF, de Sousa Fortes L, Barbosa BT, Feitosa Júnior JVA, Ribeiro CHT, da Silva Neto LV (2020) Home-based exercise during confinement in COVID-19 pandemic and mental health in adults: a cross-sectional comparative study. Revista Brasileira de Atividade Física \& Saúde. https://doi.org/10.12820/rbafs.25e0120

33. Sebastião E, Chodzko-zajko W, Schwingel A, Gobbi LTB, Papini CB, Nakamura PM et al (2013) Perceived barriers to leisure time physical activity: what Brazilians have to say ? *. Open J Prev Med 3(8):491-499. https://doi.org/10.4236/ojpm.2013.38066

34. Gilbert AS, Duncan DD, Beck AM, Eyler AA, Brownson RC (2019) A qualitative study identifying barriers and facilitators of physical activity in rural communities. J Environ Public Health. https://doi.org/10.1155/2019/7298692

Publisher's Note Springer Nature remains neutral with regard to jurisdictional claims in published maps and institutional affiliations. 